INPLASY

PROTOCOL

To cite: Ding et al. Systematic Review and Meta-analysis of 10 and 14 days Bismuthcontaining Quadruple Therapy for Helicobacter Pylori Eradication. Inplasy protocol 2021120034. doi:

10.37766/inplasy2021.12.0034

Received: 06 December 2021

Published: 06 December 2021

Corresponding author:

Xiuli Zuo

xiulizuo@163.com

Author Affiliation:

Shandong University

Support: None.

Review Stage at time of this submission: Preliminary searches.

Conflicts of interest:

None declared.

\section{Systematic Review and Meta-analysis of 10 and 14 days Bismuth-containing Quadruple Therapy for Helicobacter Pylori Eradication}

Ding, Y1; Li, Y2; Zuo, X³.

Review question / Objective: Is a 10-day course of bismuthcontaining quadruple therapy to eradicate Helicobacter pylori equivalent to a 14-day course of treatment? Are the adverse reactions of the 10-day treatment course of bismuthcontaining quadruple therapy less than that of the 14-day treatment course? A systematic review and Meta analysis of Randomized Controlled trials.

Information sources: The following bibliographic data bases were searched: PubMed, EMBASE, Web of Science, Cochrane Central Register of Controlled Trials (until the third quarter of 2021, English). Reviews, case reports, editorials, letter to the editors, and abstracts from conferences will be excluded.

INPLASY registration number: This protocol was registered with the International Platform of Registered Systematic Review and Meta-Analysis Protocols (INPLASY) on 06 December 2021 and was last updated on 06 December 2021 (registration number INPLASY2021120034).

\section{INTRODUCTION}

Review question / Objective: Is a 10-day course of bismuth-containing quadruple therapy to eradicate Helicobacter pylori equivalent to a 14-day course of treatment? Are the adverse reactions of the 10-day treatment course of bismuth-containing quadruple therapy less than that of the 14day treatment course? A systematic review and Meta analysis of Randomized Controlled trials.

Condition being studied: Helicobacter pylori (H.pylori) infection is a common global infectious disease and an important cause of diseases such as duodenal ulcer, gastric ulcer, gastric adenocarcinoma and gastric lymphoma. Because Helicobacter pylori was a notable cause of gastric 
cancer development, it should be eradicated to prevent gastric cancer. In 2015, the estimated prevalence of $\mathbf{H}$. pylori in China was $55.8 \%$. Amoxicillin-containing bismuth quadruple therapy for 14 days is the main eradication program recommended by the current guidelines. Guidelines for the management of Helicobacter pylori infection in Japan recommend a 1-week triple therapy consisting of potassium-competitive acid blocker (P-CAB), amoxicillin, and clarithromycin. Mass eradication of Helicobacter pylori has been proposed as a means to eliminate gastric cancer, And indeed reduced gastric cancer incidence with no increase in the likelihood of adverse consequences. However, Economic models have suggested that population screening and treatment for Helicobacter pylori may be cost-effective and may be the first cost-neutral screening program. If the cost of treatment can be further reduced, the benefit-cost ratio of large-scale screening for Helicobacter pylori may be improved.

\section{METHODS}

Participant or population: Inclusion criteria:1. Parallel group randomised controlled trials (RCTs)2. Trials containing bismuth-based quadruple therapy regimen (PPI bismuth-based quadruple therapy: PPI + bismuth salt (colloidal bismuth subcitrate (CBS) or bismuth subsalicylate (BSS)) + two antibiotics.); 3. Writing in English; 4. recruited individuals diagnosed as positive for $\mathrm{H}$. pylori, with at least one confirmatory test, on the basis of a rapid urease test (RUT), histology or culture of an endoscopic biopsy sample, urea breath test (UBT), or monoclonal stool antigen tests (HpSA), regardless of the presenting symptoms and disease.5. The participants must have been adults (aged 18 years or over). If the study did not specify it was for paediatric patients and we could not obtain additional data from authors, we judged that the majority of the study participants were adults, based on the following a priori criteria.6. Reporting intention to treat (ITT).7. Eradication assessments at least 4 weeks after completion of treatment. Excluded criteria:1. Trials with participants who had previously been treated with an eradication therapy;2. Reviews, case reports, editorials, letter to the editors, and abstracts from conferences. 3. Duplicate papers or evaluating the same sample.4. Studies without full-text report.Inclusion criteria:1. Parallel group randomised controlled trials (RCTs)2. Trials containing bismuth-based quadruple therapy regimen (PPI bismuth-based quadruple therapy: PPI + bismuth salt (colloidal bismuth subcitrate (CBS) or bismuth subsalicylate (BSS)) + two antibiotics.); 2. Writing in English; 3. recruited individuals diagnosed as positive for $\mathbf{H}$. pylori, with at least one confirmatory test, on the basis of a rapid urease test (RUT), histology or culture of an endoscopic biopsy sample, urea breath test (UBT), or monoclonal stool antigen tests (HpSA), regardless of the presenting symptoms and disease.4. The participants must have been adults (aged 18 years or over). If the study did not specify it was for paediatric patients and we could not obtain additional data from authors, we judged that the majority of the study participants were adults, based on the following a priori criteria.5. Reporting intention to treat (ITT).6. Eradication assessments at least 4 weeks after completion of treatment. Excluded criteria:1. Trials with participants who had previously been treated with an eradication therapy;2. Reviews, case reports, editorials, letter to the editors, and abstracts from conferences. Duplicate papers or evaluating the same sample.3. Studies without full-text report.

Intervention: A 10-day course of PPI bismuth-based quadruple Helicobacter pylori eradication treatment. (PPI bismuthbased quadruple therapy: PPI + bismuth salt (colloidal bismuth subcitrate (CBS) or bismuth subsalicylate (BSS)) + two antibiotics.)

Comparator: A 14-day course of PPI bismuth-based quadruple Helicobacter pylori eradication treatment. (PPI bismuthbased quadruple therapy: PPI + bismuth salt (colloidal bismuth subcitrate (CBS) or 
bismuth subsalicylate (BSS)) + two antibiotics.)

Study designs to be included: We considered only parallel group randomised controlled trials (RCTs) eligible for inclusion in the review.

Eligibility criteria: Inclusion criteria:1. Parallel group randomised controlled trials (RCTs)2. Trials containing bismuth-based quadruple therapy regimen (PPI bismuthbased quadruple therapy: PPI + bismuth salt (colloidal bismuth subcitrate (CBS) or bismuth subsalicylate (BSS)) + two antibiotics.); 3 . Writing in English; 4. recruited individuals diagnosed as positive for $\mathrm{H}$. pylori, with at least one confirmatory test, on the basis of a rapid urease test (RUT), histology or culture of an endoscopic biopsy sample, urea breath test (UBT), or monoclonal stool antigen tests (HpSA), regardless of the presenting symptoms and disease.5. The participants must have been adults (aged 18 years or over). If the study did not specify it was for paediatric patients and we could not obtain additional data from authors, we judged that the majority of the study participants were adults, based on the following a priori criteria.6. Reporting intention to treat (ITT).7. Eradication assessments at least 4 weeks after completion of treatment. Excluded criteria:1. Trials with participants who had previously been treated with an eradication therapy;2. Reviews, case reports, editorials, letter to the editors, and abstracts from conferences.3. Duplicate papers or evaluating the same sample.4. Studies without full-text report.Inclusion criteria:1. Parallel group randomised controlled trials (RCTs)2. Trials containing bismuth-based quadruple therapy regimen (PPI bismuth-based quadruple therapy: PPI + bismuth salt (colloidal bismuth subcitrate (CBS) or bismuth subsalicylate (BSS)) + two antibiotics.); 2. Writing in English; 3. recruited individuals diagnosed as positive for $\mathbf{H}$. pylori, with at least one confirmatory test, on the basis of a rapid urease test (RUT), histology or culture of an endoscopic biopsy sample, urea breath test (UBT), or monoclonal stool antigen tests (HpSA), regardless of the presenting symptoms and disease.4. The participants must have been adults (aged 18 years or over). If the study did not specify it was for paediatric patients and we could not obtain additional data from authors, we judged that the majority of the study participants were adults, based on the following a priori criteria.5. Reporting intention to treat (ITT).6. Eradication assessments at least 4 weeks after completion of treatment. Excluded criteria:1. Trials with participants who had previously been treated with an eradication therapy;2. Reviews, case reports, editorials, letter to the editors, and abstracts from conferences.Duplicate papers or evaluating the same sample.3. Studies without full-text report.

Information sources: The following bibliographic data bases were searched: PubMed, EMBASE, Web of Science, Cochrane Central Register of Controlled Trials (until the third quarter of 2021, English). Reviews, case reports, editorials, letter to the editors, and abstracts from conferences will be excluded.

Main outcome(s): The eradication rate of 14 versus 7 days of different regimens in eradicating $H$. pylori. (Eradication rate: The percentage of successful eradication of all participants.)

Additional outcome(s): The incidence of adverse effects associated with different durations of $\mathbf{H}$. pylori eradication therapy. (adverse effects: the proportion of all participants who have an adverse reaction.)

Quality assessment / Risk of bias analysis: Two reviewers will independently assess risk of bias based on the following domains from recommendations from the Cochrane handbook: 1. Adequate sequence generation; 2. Allocation concealment; 3. Blinding; 4. Incomplete outcome data and how it was addressed; 5. Selective reporting of the outcome; 6. Any other biases. results of bias assessment will be presented in a figure and a graph indicating low, high or unclear risk of bias for each of the 6 items in each trial. Sensitivity analysis will be conducted based on the bias 
assessment to assess robustness of results.

Strategy of data synthesis: Relative Risk (RR) and its 95\% confidence intervals (95\% CI) will be calculated for the outcome eradication rate in each included trial based on intention to treat (ITT). Due to expected heterogeneity among the trials, Meta-analysis using the random-effects model will be conducted to pool RR.

Subgroup analysis: The subgroup analyses will also be undertaken to assess if the different antibiotics groups produce different treatment effects.

Sensitivity analysis: Sensitivity analysis to assess robustness of results and subgroup analysis to determine whether the summary effects vary in relation to clinical characteristics of the trials included are pre-specified. In addition, sensitivity analysis, including only those trials free of any assessed bias will be conducted.

Country(ies) involved: China.

Keywords: Helicobacter pylori; duration; bismuth

Contributions of each author:

Author 1 - YUMING DING.

Email: dingyuming@mail.sdu.edu.cn

Author 2 - XIULI ZUO.

Email: xiulizuo@163.com

Author 3 - YUEYUE LI. 